\title{
RELATIONS AMONG DIRICHLET SERIES WHOSE COEFFICIENTS ARE CLASS NUMBERS OF BINARY CUBIC FORMS II
}

\author{
Yasuo Ohno and Takashi TANiguchi
}

\begin{abstract}
As a continuation of the authors' and Wakatsuki's previous paper [5], we study relations among Dirichlet series whose coefficients are class numbers of binary cubic forms. We show that, for all integral models of the space of binary cubic forms, the associated Dirichlet series satisfy self-dual identities.
\end{abstract}

\section{Introduction}

The theory of zeta functions for the space of binary cubic forms was initiated by Shintani [7] as a fine example of zeta functions of prehomogeneous vector spaces [6]. He introduced 4 Dirichlet series $\xi_{1,1}(s), \xi_{1,2}(s), \xi_{1,1}^{*}(s), \xi_{1,2}^{*}(s)$ whose coefficients are class numbers of integral binary cubic forms, and established that they have remarkable analytic properties. These 4 zeta functions are associated with the "standard" integral models, and our purpose in this paper is to study the zeta functions for all integral models.

Let us recall the definition of the zeta function. Let $V_{\mathbb{Q}}$ be the space of binary cubic forms over the rational number field $\mathbb{Q}$;

$$
V_{\mathbb{Q}}:=\left\{x(u, v)=a u^{3}+b u^{2} v+c u v^{2}+d v^{3} \mid a, b, c, d \in \mathbb{Q}\right\} .
$$

We express elements of $V_{\mathbb{Q}}$ as $x=x(u, v)=a u^{3}+b u^{2} v+c u v^{2}+d v^{3}$. We identify $V_{\mathbb{Q}}$ with $\mathbb{Q}^{4}$ via $V_{\mathbb{Q}} \ni x \mapsto(a, b, c, d) \in \mathbb{Q}^{4}$, which allows us to simply write $x=(a, b, c, d)$. Let $P(x)$ be the discriminant of $x \in V_{\mathbb{Q}}$ :

$$
P(x):=\operatorname{Disc}(x(u, v))=b^{2} c^{2}+18 a b c d-4 a c^{3}-4 b^{3} d-27 a^{2} d^{2} .
$$

The group $\mathrm{SL}_{2}(\mathbb{Z})$ acts on $V_{\mathbb{Q}}$ by the linear change of variables, and the polynomial $P(x)$ is invariant under this action.

We recall the classification of $\mathrm{SL}_{2}(\mathbb{Z})$-invariant lattices in $V_{\mathbb{Q}}$. Define

$$
\begin{aligned}
& L_{1}:=\left\{x \in V_{\mathbb{Q}} \mid a, b, c, d \in \mathbb{Z}\right\}=\mathbb{Z}^{4}, \\
& L_{2}:=\left\{(a, b, c, d) \in L_{1} \mid a+b+d, a+c+d \in 2 \mathbb{Z}\right\}, \\
& L_{3}:=\left\{(a, b, c, d) \in L_{1} \mid a+b+c, b+c+d \in 2 \mathbb{Z}\right\}, \\
& L_{4}:=\left\{(a, b, c, d) \in L_{1} \mid b+c \in 2 \mathbb{Z}\right\}, \\
& L_{5}:=\left\{(a, b, c, d) \in L_{1} \mid a, d, b+c \in 2 \mathbb{Z}\right\},
\end{aligned}
$$

Received by the editors November 9, 2011. 
and

$$
\begin{aligned}
& L_{1}^{*}:=\left\{x \in V_{\mathbb{Q}} \mid a, d \in \mathbb{Z}, b, c \in 3 \mathbb{Z}\right\}, \\
& L_{2}^{*}:=L_{1}^{*} \cap L_{3}, \quad L_{3}^{*}:=L_{1}^{*} \cap L_{2}, \quad L_{4}^{*}:=L_{1}^{*} \cap L_{5}, \quad L_{5}^{*}:=L_{1}^{*} \cap L_{4} .
\end{aligned}
$$

We can easily check that $L_{4} \supset L_{2}, L_{4} \supset L_{5}$ and $L_{3} \supset L_{5}$. Similarly, we have $L_{5}^{*} \supset L_{3}^{*}$, $L_{5}^{*} \supset L_{4}^{*}$ abd $L_{2}^{*} \supset L_{4}^{*}$.
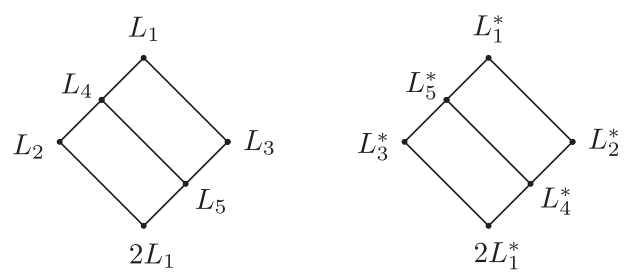

The authors and Wakatsuki [5] showed that, up to $\mathbb{Q}^{\times}$-multiplication, this is a complete list of $\mathrm{SL}_{2}(\mathbb{Z})$-invariant lattices in $V_{\mathbb{Q}}$. Hence there are 10 different integral models of $V_{\mathbb{Q}}$.

The zeta functions are defined as follows.

Definition 1.1. For $1 \leq i \leq 5$ and $1 \leq j \leq 2$, we define

$$
\begin{aligned}
\xi_{i, j}(s) & :=\sum_{\substack{x \in \mathrm{SL}_{2}(\mathbb{Z}) \backslash L_{i} \\
(-1)^{j-1} P(x)>0}} \frac{|\operatorname{Stab}(x)|^{-1}}{|P(x)|^{s}}, \\
\xi_{i, j}^{*}(s):= & \sum_{\substack{x \in \mathrm{SL}_{2}(\mathbb{Z}) \backslash L_{i}^{*} \\
(-1)^{j-1} P(x)>0}} \frac{|\operatorname{Stab}(x)|^{-1}}{|P(x) / 27|^{s}},
\end{aligned}
$$

and call them the zeta functions associated with $L_{i}$ and $L_{i}^{*}$, respectively. Here $|\operatorname{Stab}(x)|$ is the order of stabilizers of $x$ in $\mathrm{SL}_{2}(\mathbb{Z})$.

Note that $|\operatorname{Stab}(x)|$ is either 1 or 3 , and that $P(x)$ is a multiple of 27 for $x \in L_{i}^{*}$.

Shintani [7] showed that $\xi_{1, j}(s)$ and $\xi_{1, j}^{*}(s)$ have holomorphic continuations to the whole complex plane except for simple poles at $s=1,5 / 6$, and satisfy a functional equation. He also computed the residues explicitly. In [5], we proved similar analytic properties for $2 \leq i \leq 5$.

One other significant property of $\xi_{1, j}(s)$ and $\xi_{1, j}^{*}(s)$ was discovered about 25 years after Shintani's work. The following identities were conjectured by the first author [4] and subsequently proved by Nakagawa [3].

Theorem 1.2 (Conjectured in [4], proved in [3]). For the zeta functions associated with $L_{1}$ and $L_{1}^{*}$, we have the identities

$$
\xi_{1,1}^{*}(s)=\xi_{1,2}(s), \quad \xi_{1,2}^{*}(s)=3 \xi_{1,1}(s) .
$$

Although the identities (1.1) are rather simple, no elementary proof of this theorem is known. Indeed, Nakagawa proved them as a consequence of the sophisticated use of class field theory. As we will describe in Theorem 1.5, this theorem has an important application for functional equations. Hence it is natural to ask whether there are similar identities of the zeta functions for other integral models. Theorem 1.3 below 
presents our previous partial answer to this, while Theorem 1.4 presents our main theorem which is in the affirmative.

To state our results, it is convenient to put

$$
\xi_{i}(s):=\left(\begin{array}{l}
\xi_{i, 1}(s) \\
\xi_{i, 2}(s)
\end{array}\right), \quad \xi_{i}^{*}(s):=\left(\begin{array}{l}
\xi_{i, 1}^{*}(s) \\
\xi_{i, 2}^{*}(s)
\end{array}\right), \quad A:=\left(\begin{array}{ll}
0 & 1 \\
3 & 0
\end{array}\right)
$$

Then (1.1) is written as $\xi_{1}^{*}(s)=A \cdot \xi_{1}(s)$. For $i=2,3$, the authors and Wakatsuki proved the following in the previous paper [5].

Theorem 1.3 ( [5]). For the zeta functions associated with $L_{2}, L_{2}^{*}$ and $L_{3}, L_{3}^{*}$, we have

$$
\begin{aligned}
& \xi_{2}^{*}(s)=A \cdot \xi_{2}(s), \\
& \xi_{3}^{*}(s)=A \cdot \xi_{3}(s) .
\end{aligned}
$$

On the other hand, for $i=4,5, \xi_{i}^{*}(s)$ and $A \cdot \xi_{i}(s)$ do not coincide. These discrepancies themselves are not surprising since the indices $\left[L_{1}: L_{i}\right]$ and $\left[L_{1}^{*}: L_{i}^{*}\right]$ do not coincide.

In this paper we study these remaining cases. In particular, we prove identities like (1.1), (1.2) for $i=4,5$ in certain linear combinations of the zeta functions. The following is the main result of this paper.

Theorem 1.4 (Main Theorem). For linear combinations

$$
\begin{aligned}
\theta(s) & :=\xi_{1}(s)-2 \xi_{3}(s)-\xi_{4}(s)+4 \xi_{5}(s), \\
\eta(s) & :=2^{2 s}\left(\xi_{4}(s)-\xi_{2}(s)-\xi_{5}(s)+2^{1-4 s} \xi_{1}(s)\right), \\
\theta^{*}(s) & :=2^{2 s}\left(\xi_{5}^{*}(s)-\xi_{3}^{*}(s)-\xi_{4}^{*}(s)+2^{1-4 s} \xi_{1}^{*}(s)\right), \\
\eta^{*}(s) & :=\xi_{1}^{*}(s)-2 \xi_{2}^{*}(s)-\xi_{5}^{*}(s)+4 \xi_{4}^{*}(s),
\end{aligned}
$$

we have the identities

$$
\begin{aligned}
& \theta^{*}(s)=A \cdot \theta(s), \\
& \eta^{*}(s)=A \cdot \eta(s) .
\end{aligned}
$$

We apply these identities to the functional equations. We put

$$
\begin{aligned}
& \Delta_{+}(s):=\left(\frac{2^{4} 3^{3}}{\pi^{4}}\right)^{s / 2} \Gamma\left(\frac{s}{2}\right) \Gamma\left(\frac{s}{2}+\frac{1}{2}\right) \Gamma\left(\frac{s}{2}-\frac{1}{12}\right) \Gamma\left(\frac{s}{2}+\frac{1}{12}\right), \\
& \Delta_{-}(s):=\left(\frac{2^{4} 3^{3}}{\pi^{4}}\right)^{s / 2} \Gamma\left(\frac{s}{2}\right) \Gamma\left(\frac{s}{2}+\frac{1}{2}\right) \Gamma\left(\frac{s}{2}+\frac{5}{12}\right) \Gamma\left(\frac{s}{2}+\frac{7}{12}\right) .
\end{aligned}
$$

Then by plugging (1.1), (1.2) into the functional equations obtained by Shintani's theory [7], the followings were proved: 
Theorem $1.5([3-5])$. Let $i=1,2,3$. For each sign, define

$$
\xi_{i, \pm}(s):=\sqrt{3} \xi_{i, 1}(s) \pm \xi_{i, 2}(s)
$$

Let $a_{1}=0, a_{2}=a_{3}=2$. Then they satisfy the functional equations

$$
2^{a_{i} s} \Delta_{ \pm}(s) \xi_{i, \pm}(s)=2^{a_{i}(1-s)} \Delta_{ \pm}(1-s) \xi_{i, \pm}(1-s) .
$$

Similarly, as a consequence of (1.3), we have the following.

Theorem 1.6 (Corollary to Theorem 1.4). We define

$$
\theta_{ \pm}(s):=\sqrt{3} \theta_{1}(s) \pm \theta_{2}(s) \quad \text { and } \quad \eta_{ \pm}(s):=\sqrt{3} \eta_{1}(s) \pm \eta_{2}(s)
$$

where $\theta(s)=\left(\begin{array}{l}\theta_{1}(s) \\ \theta_{2}(s)\end{array}\right)$ and $\eta(s)=\left(\begin{array}{l}\eta_{1}(s) \\ \eta_{2}(s)\end{array}\right)$. Then

$$
\begin{aligned}
& 2^{s} \Delta_{ \pm}(s) \theta_{ \pm}(s)=2^{1-s} \Delta_{ \pm}(1-s) \theta_{ \pm}(1-s) \\
& 2^{s} \Delta_{ \pm}(s) \eta_{ \pm}(s)=2^{1-s} \Delta_{ \pm}(1-s) \eta_{ \pm}(1-s) .
\end{aligned}
$$

Hence the functional equations of the zeta functions can be expressed in self-dual forms for all integral models. In view of (1.4) and (1.5), we may say that the "conductor" of the Dirichlet series $\xi_{1, \pm}(s), \xi_{2, \pm}(s), \xi_{3, \pm}(s), \theta_{ \pm}(s)$ and $\eta_{ \pm}(s)$ are $2^{4} 3^{3}, 2^{8} 3^{3}$, $2^{8} 3^{3}, 2^{6} 3^{3}$ and $2^{6} 3^{3}$, respectively ${ }^{1}$. Their poles and residues are described as follows.

Theorem 1.7. The Dirichlet series $\xi_{1,+}(s), \xi_{2,+}(s), \xi_{3,+}(s), \theta_{+}(s)$ and $\eta_{+}(s)$ are holomprhic except for simple poles at $s=1$ and $s=5 / 6$, while $\xi_{1,-}(s), \xi_{2,-}(s)$, $\xi_{3,-}(s), \theta_{-}(s)$ and $\eta_{-}(s)$ are holomorphic except for a simple pole at $s=1$. Their residues are given as follows:

\begin{tabular}{l|ccccc}
\hline & $\xi_{1,+}(s)$ & $\xi_{2,+}(s)$ & $\xi_{3,+}(s)$ & $\theta_{+}(s)$ & $\eta_{+}(s)$ \\
\hline Residue at $s=1$ & $\frac{2 \sqrt{3}+3}{18} \pi^{2}$ & $\frac{2 \sqrt{3}+3}{72} \pi^{2}$ & $\frac{2 \sqrt{3}+3}{72} \pi^{2}$ & $\frac{7 \sqrt{3}+9}{72} \pi^{2}$ & $\frac{5 \sqrt{3}+9}{72} \pi^{2}$ \\
Residue at $s=\frac{5}{6}$ & $\frac{\Gamma(1 / 3)^{3}}{3 \pi} \zeta\left(\frac{1}{3}\right)$ & $\frac{\Gamma(1 / 3)^{3}}{12 \pi} \zeta\left(\frac{1}{3}\right)$ & $\frac{\Gamma(1 / 3)^{3}}{12 \pi} \zeta\left(\frac{1}{3}\right)$ & $\frac{\Gamma(1 / 3)^{3}}{3 \sqrt[3]{2} \pi} \zeta\left(\frac{1}{3}\right)$ & $\frac{\Gamma(1 / 3)^{3}}{3 \sqrt[3]{2} \pi} \zeta\left(\frac{1}{3}\right)$ \\
\hline "Conductor" & $2^{4} 3^{3}$ & $2^{8} 3^{3}$ & $2^{8} 3^{3}$ & $2^{6} 3^{3}$ & $2^{6} 3^{3}$ \\
\hline \hline & $\xi_{1,-}(s)$ & $\xi_{2,-}(s)$ & $\xi_{3,-}(s)$ & $\theta_{-}(s)$ & $\eta_{-}(s)$ \\
\hline Residue at $s=1$ & $\frac{2 \sqrt{3}-3}{18} \pi^{2}$ & $\frac{2 \sqrt{3}-3}{72} \pi^{2}$ & $\frac{2 \sqrt{3}-3}{72} \pi^{2}$ & $\frac{7 \sqrt{3}-9}{72} \pi^{2}$ & $\frac{5 \sqrt{3}-9}{72} \pi^{2}$ \\
\hline "Conductor" & $2^{4} 3^{3}$ & $2^{8} 3^{3}$ & $2^{8} 3^{3}$ & $2^{6} 3^{3}$ & $2^{6} 3^{3}$ \\
\hline
\end{tabular}

An interesting phenomenon is that the latter 5 Dirichlet series are holomorphic at $s=5 / 6$.

In the previous paper [5], we proved Theorem 1.3 by reduction to Theorem 1.2. In this paper, we prove Theorem 1.4 also by reduction to Theorem 1.2. However we

\footnotetext{
${ }^{1}$ All of these 10 Dirichlet series are of the form $\sum_{n \geq 1} a_{n} / n^{s}$, and by using the table of the coefficients in [5], we can confirm that the greatest common divisor of $\left\{n \mid a_{n} \neq 0\right\}$ is 1 .
} 
need to argue more carefully since the relations between $\xi_{4}(s), \xi_{5}(s)$ and $\xi_{1}(s)$ are not as direct as those of $\xi_{2}(s), \xi_{3}(s)$ and $\xi_{1}(s)$. Our idea is to look at certain subsets of $L_{1}$ which are no longer $\mathrm{SL}_{2}(\mathbb{Z})$-invariant but invariant under certain congruence subgroups such as $\Gamma_{0}(2)$ or $\Gamma(2)$. We study them in terms of the induction in the category of $G$-sets where $G$ is a group. The zeta functions behave quite well with respect to this induction (Proposition 3.2), and this enables us to bring $\xi_{4}(s), \xi_{5}(s)$ and $\xi_{1}(s)$ into connection. We note that in a recent paper of the second author and Thorne [8], similar self-dual identities were found also for certain divisible zeta functions.

We mention that important algebraic interpretations of integer orbits of $L_{1}$ and $L_{1}^{*}$ are known. Precisely, $\mathrm{GL}_{2}(\mathbb{Z}) \backslash L_{1}$ has a canonical bijection to the set of isomorphism classes of cubic rings, while $\mathrm{SL}_{2}(\mathbb{Z}) \backslash L_{1}^{*}$ parametrizes 3 -torsions in the ideal class groups of quadratic rings. Among other applications, these interpretations were key ingredients for Nakagawa's proof of Theorem 1.2 in terms of class field theory. Such algebraic interpretations of integer orbits for many other prehomogeneous vector spaces were systematically discovered in Bhargava's groundbreaking work of higher composition laws [1]. Taking these results into consideration, we expect that there might exist interesting interpretations for integer orbits of $L_{i}, L_{i}^{*}$ for $2 \leq i \leq 5$ also. We hope the theory of integer orbits will be pursued further in the future.

This paper is organized as follows. In Section 2, we introduce the notion of induction in our setting. After that, we study the set

$\left\{x \in L_{1} \mid P(x) \equiv l \quad \bmod 32\right\}$ and $\left\{x \in L_{1}^{*} \mid P(x) / 27 \equiv-l \quad \bmod 32\right\} \quad($ for $l=4,20)$

in some detail. In Propositions 2.4 and 2.7, we prove that the actions of $\mathrm{SL}_{2}(\mathbb{Z})$ on these sets are induced from the action of $\Gamma_{0}(2)$ on certain of their subsets. The proof of Theorem 1.4 is given in Section 3. In Theorem 3.8, we express the partial zeta functions associated with the sets just above as linear combinations of $\xi_{i}(s)$ or $\xi_{i}^{*}(s)$. This enables us to reduce Theorem 1.4 to Theorem 1.2. In Section 4, we prove Theorems 1.6 and 1.7.

Notations. The notations introduced in this section are used throughout this paper. For a finite set $X$, its cardinality is denoted by $|X|$. If a group $G$ acts on a set $X$, then for $x \in X$ we put $G_{x}=\{g \in G \mid g x=x\}$. In this paper we frequently consider congruences in $\mathbb{Z}$. If $a-a^{\prime} \in N \mathbb{Z}$ then we write $a \equiv a^{\prime}(N)$ as well as $a \equiv a^{\prime} \bmod N$. For $a, a^{\prime}, a^{\prime \prime}, \ldots \in \mathbb{Z}, " a \bmod N \equiv a^{\prime} \equiv a^{\prime \prime} \equiv \cdots "$ means $a-a^{\prime}, a^{\prime}-a^{\prime \prime}, \ldots \in N \mathbb{Z}$.

We use the following standard notation for the congruence subgroups of $\mathrm{SL}_{2}(\mathbb{Z})$ :

$$
\begin{aligned}
\Gamma(N) & =\left\{\left(\begin{array}{ll}
p & q \\
r & s
\end{array}\right) \in \mathrm{SL}_{2}(\mathbb{Z}) \mid\left(\begin{array}{ll}
p & q \\
r & s
\end{array}\right) \equiv\left(\begin{array}{ll}
1 & 0 \\
0 & 1
\end{array}\right) \quad \bmod N\right\}, \\
\Gamma_{0}(N) & =\left\{\left(\begin{array}{ll}
p & q \\
r & s
\end{array}\right) \in \mathrm{SL}_{2}(\mathbb{Z}) \mid r \equiv 0(N)\right\}, \\
\Gamma^{0}(N) & =\left\{\left(\begin{array}{ll}
p & q \\
r & s
\end{array}\right) \in \mathrm{SL}_{2}(\mathbb{Z}) \mid q \equiv 0(N)\right\} .
\end{aligned}
$$

Note that $\Gamma(1)=\mathrm{SL}_{2}(\mathbb{Z})$. Finally, we put

$$
\mathcal{E}:=2 \mathbb{Z} \quad \text { and } \quad \mathcal{O}:=2 \mathbb{Z}+1,
$$

the set of even integers and odd integers, respectively. 


\section{Expressions in induced forms}

To prove the main theorem, we use the notion of "induction" in the category of $G$-sets. For the convenience of the reader, we summarize its definition and basic properties here. The formulation is very similar to the induction of representations of finite groups. We omit the elementary proofs of the basic facts.

Let $G$ be a group. Assume that its subgroup $H$ acts on a set $Y$. Then, up to equivalence, there exists a unique pair $(\iota, \widetilde{Y})$ where $\widetilde{Y}$ is a $G$-set and $\iota: Y \hookrightarrow \widetilde{Y}$ is an injective $H$-map which satisfies the following conditions;

(1) the map $\tilde{\iota}: H \backslash Y \rightarrow G \backslash \widetilde{Y}$ induced from $\iota$ is bijective, and

(2) for all $y \in Y, H_{y}=G_{\iota(y)}$.

The pair $(\iota, \tilde{Y})$ is constructed as follows: Consider an equivalence relation $\sim$ on $G \times Y$ so that $(g, y) \sim\left(g^{\prime}, y^{\prime}\right)$ if and only if there exists $h \in H$ such that $g^{\prime}=g h^{-1}$ and $y^{\prime}=h y$. Let $\tilde{Y}$ be the quotient set of $G \times Y$ under this equivalence relation. The canonical image of $(g, y) \in G \times Y$ in $\tilde{Y}$ is again denoted by $(g, y)$. The map $G \times \tilde{Y} \ni$ $\left(g^{\prime},(g, x)\right) \mapsto\left(g^{\prime} g, x\right) \in \widetilde{Y}$ is well defined, and this defines an action of $G$ on $\tilde{Y}$. Let $\iota: Y \ni y \mapsto(e, y) \in \widetilde{Y}$, where $e \in G$ is the identity. Then the pair $(\iota, \tilde{Y})$ satisfies the desired properties. Let us write this $\tilde{Y}$ as $G \times_{H} Y$.

Let $X$ be a $G$-set, and assume that a subset $Y \subset X$ is invariant under the action of a subgroup $H \subset G$. Then we have a natural $G$-map $G \times_{H} Y \ni(g, y) \mapsto g y \in X$ between $G$-sets. When this map is bijective, we write $X=G \times_{H} Y$ and say that $(G, X)$ is induced from $(H, Y)$. For this condition, we have the following criteria.

Lemma 2.1. Let $G, H$ and $X, Y$ be as above. Then $X=G \times_{H} Y$ if and only if

(1) the map $G \times Y \ni(g, y) \rightarrow g y \in X$ is surjective, and

(2) for $y \in Y$ and $g \in G, g y \in Y$ if and only if $g \in H$.

The following assertion immediately follows from this lemma.

Lemma 2.2. If $X^{\prime}$ is a $G$-invariant subset of $G \times_{H} Y$, then $X^{\prime}=G \times_{H}\left(Y \cap X^{\prime}\right)$.

If $(G, X)$ is induced from $(H, Y)$, then there is a natural bijection between the sets of orbits $G \backslash X$ and $H \backslash Y$, and the structures of the stabilizers are preserved under the correspondence. The role of this notion of induction in the theory of the zeta functions will become apparent in Section 3 (see Proposition 3.2).

Remark 2.3. In the terminology of category theory, the morphism $\{H$-set $\} \ni Y \mapsto$ $G \times{ }_{H} Y \in\{G$-set $\}$ is the left adjoint functor of the restriction functor $\{G$-set $\} \ni X \mapsto$ $X \in\{H$-set $\}$, i.e., $\operatorname{Hom}_{H}(Y, X) \cong \operatorname{Hom}_{G}\left(G \times_{H} Y, X\right)$.

We now consider the space of binary cubic forms. For $l, N \in \mathbb{Z}$, we define

$$
L_{1}^{\equiv l(N)}:=\left\{x \in L_{1} \mid P(x) \equiv l(N)\right\} .
$$

The primary purpose of this section is to prove the following. 
Proposition 2.4. Define

$$
\begin{aligned}
& X_{1}=\left\{(a, b, c, d) \in \mathbb{Z}^{4} \mid b \in \mathcal{O}, c \in 2 \mathcal{O}, d \in 4 \mathcal{E}\right\}, \\
& X_{2}=\left\{(a, b, c, d) \in \mathbb{Z}^{4} \mid b \in \mathcal{O}, c \in 2 \mathcal{O}, d \in 4 \mathcal{O}\right\}, \\
& X_{3}=\left\{(a, b, c, d) \in \mathbb{Z}^{4} \mid a \in \mathcal{O}, b, c \in \mathcal{E}, d \in 2 \mathcal{O}\right\} .
\end{aligned}
$$

Then

$$
\begin{aligned}
L_{1}^{\equiv 4(32)} & =\Gamma(1) \times_{\Gamma_{0}(2)} X_{1}, \\
L_{1}^{\equiv 20(32)} & =\Gamma(1) \times_{\Gamma_{0}(2)}\left(X_{2} \sqcup X_{3}\right) .
\end{aligned}
$$

To prove this proposition, we describe when $P(x) \equiv 4(32)$ and $P(x) \equiv 20(32)$ in terms of the conditions of the coordinates $a, b, c, d$ of $x$. This is given in Lemma 2.6, and after that we will prove this proposition.

We begin by determining when $P(x) \equiv 4$ (16) holds.

Lemma 2.5. For $x=(a, b, c, d) \in \mathbb{Z}^{4}, P(x) \equiv 4$ (16) if and only if one of the following conditions is satisfied;

(1) $a, b, c, d \in \mathcal{O}, a+b+c+d \in 2 \mathcal{O}$,

(2) $b, c \in \mathcal{E}, a d \in 2 \mathcal{O}$,

(3) $a \in 2 \mathcal{E}, b \in 2 \mathcal{O}, c \in \mathcal{O}$,

(4) $d \in 2 \mathcal{E}, c \in 2 \mathcal{O}, b \in \mathcal{O}$,

(5) $b+c \in \mathcal{O}, a+c, b+d \in 2 \mathcal{E}$.

Proof. We write $P=P(x)$. We have $P=(a d+b c)^{2}+4 R+16\left(a b c d-2 a^{2} d^{2}\right)$, where

$$
R=a^{2} d^{2}-a c^{3}-b^{3} d
$$

Hence

$$
P \equiv(a d+b c)^{2}+4 R(16) .
$$

If $a d+b c$ is odd, then so is $P$. Hence in what follows we assume that $a d+b c$ is even, and consider when $P \equiv 4$ (16) holds. Note that in this case $(a d+b c)^{2} \equiv 0(16)$ or $\equiv 4$ (16) when $a d+b c \in 2 \mathcal{E}$ or $a d+b c \in 2 \mathcal{O}$, respectively. We also note that if $n$ is odd then $n^{2} \equiv 1(8)$.

Since we assume that $a d+b c$ is even, the parity of $a d$ and $b c$ coincide. Suppose both $a d$ and $b c$ are odd. Then $a, b, c, d \in \mathcal{O}$ and so $R \bmod 4 \equiv 1-a c-b d \equiv 1,3$. Hence $P \equiv 4$ (16) if and only if $a d+b c \in 2 \mathcal{E}$ and $1-a c-b d \in 4 \mathbb{Z}+1$. Under the conditions $a, b, c, d \in \mathcal{O}$, this is equivalent to $a+b+c+d \in 2 \mathcal{O}$. This is condition (1).

For the rest of the proof, we assume that both $a d$ and $b c$ are even. In this case $R$ $\bmod 4 \equiv-a c^{3}-b^{3} d$. First assume $b, c \in \mathcal{E}$. Then $R \equiv 0$ (4) and hence $P \equiv 4$ (16) if and only if $a d+b c \in 2 \mathcal{O}$. Hence $a d \in 2 \mathcal{O}$ and we get the condition (2). Next assume $b \in \mathcal{E}$ and $c \in \mathcal{O}$. Since $R \equiv-a c(4), P \equiv 4$ (16) if and only if either (i) $a d+b c \in 2 \mathcal{O}, a c \in 4 \mathbb{Z}$ or (ii) $a d+b c \in 2 \mathcal{E}, a c+1 \in 4 \mathbb{Z}$. In the case (i), since $c \in \mathcal{O}$, we have $a \in 2 \mathcal{E}$ and so $a d+b c \in 2 \mathcal{O}$ if and only if $b \in 2 \mathcal{O}$. This is condition (3). In the case (ii), since $a \in \mathcal{O}$, we have $d \in \mathcal{E}$. Under the conditions $a, c \in \mathcal{O}, b, d \in \mathcal{E}$, (ii) hold if and only if $a+c, b+d \in 2 \mathcal{E}$. Hence we get the condition (A) : $b \in \mathcal{E}, c \in \mathcal{O}, a+c, b+d \in 2 \mathcal{E}$. Finally we assume $b \in \mathcal{O}$ and $c \in \mathcal{E}$. By the same argument, $P \equiv 4$ (16) happens exactly when either (4) or (B) : $b \in \mathcal{O}, c \in \mathcal{E}, a+c, b+d \in 2 \mathcal{E}$ are satisfied. Since "either (A) or (B)" are equivalent to the condition (5), we have the lemma. 
Using this lemma, we have the following assertion which describes when $P(x) \equiv$ 4,20 (32) happens in terms of conditions of $a, b, c, d$.

Lemma 2.6. We put

$$
\begin{aligned}
X_{1}^{\prime} & =\left\{(a, b, c, d) \in \mathbb{Z}^{4} \mid c \in \mathcal{O}, b \in 2 \mathcal{O}, a \in 4 \mathcal{E}\right\}, \\
X_{1}^{\prime \prime} & =\left\{(a, b, c, d) \in \mathbb{Z}^{4} \mid b+c \in \mathcal{O}, a+c \in 2 \mathcal{E}, a+b+c+d \in 4 \mathcal{E}\right\}, \\
X_{2}^{\prime} & =\left\{(a, b, c, d) \in \mathbb{Z}^{4} \mid c \in \mathcal{O}, b \in 2 \mathcal{O}, a \in 4 \mathcal{O}\right\}, \\
X_{2}^{\prime \prime} & =\left\{(a, b, c, d) \in \mathbb{Z}^{4} \mid b+c \in \mathcal{O}, a+c \in 2 \mathcal{E}, a+b+c+d \in 4 \mathcal{O}\right\}, \\
X_{3}^{\prime} & =\left\{(a, b, c, d) \in \mathbb{Z}^{4} \mid d \in \mathcal{O}, b, c \in \mathcal{E}, a \in 2 \mathcal{O}\right\}, \\
X_{3}^{\prime \prime} & =\left\{(a, b, c, d) \in \mathbb{Z}^{4} \mid a, b, c, d \in \mathcal{O}, a+b+c+d \in 2 \mathcal{O}\right\} .
\end{aligned}
$$

Then

$$
\begin{aligned}
L_{1}^{\equiv 4(32)} & =X_{1} \sqcup X_{1}^{\prime} \sqcup X_{1}^{\prime \prime}, \\
L_{1}^{\equiv 20(32)} & =X_{2} \sqcup X_{2}^{\prime} \sqcup X_{2}^{\prime \prime} \sqcup X_{3} \sqcup X_{3}^{\prime} \sqcup X_{3}^{\prime \prime} .
\end{aligned}
$$

Proof. If $P(x) \equiv 4$ (16), then either $P(x) \equiv 4(32)$ or $P(x) \equiv 20$ (32). Hence we can prove this lemma by examining each of five cases listed in Lemma 2.5. Since the argument is elemental and simple, we briefly sketch the outline of the proof. Recall that

$$
P=(a d+b c)^{2}+4 R+16\left(a b c d-2 a^{2} d^{2}\right) .
$$

In case (1), since $R=a^{2} d^{2}-a c^{3}-b^{3} d \equiv 1-(a c+b d)(8)$, we have $P \bmod 32 \equiv$ $(a d+b c)^{2}-4(a c+b d)+20$. We note that $a d+b c, a c+b d \in 2 \mathcal{E}$ (see the proof of the previous lemma). Moreover, $a d+b c+a c+b d=(a+b)(c+d) \in 8 \mathbb{Z}$ since $a+b, c+d \in \mathcal{E}$ and $a+b+c+d \in 2 \mathcal{O}$. Hence $P \equiv 20$ (32). This case corresponds to $X_{3}^{\prime \prime}$.

In case $(2)$, since $a d+b c \in 2 \mathcal{O},(a d+b c)^{2} \equiv 4(32)$. Also $R \equiv 4(8)$, and therefore $P \equiv 20$ (32). This case corresponds to $X_{3}$ and $X_{3}^{\prime}$. In case (3), again we have $a d+$ $b c \in 2 \mathcal{O}$ and hence $(a d+b c)^{2} \equiv 4(32)$. Moreover, $R \bmod 8 \equiv-a c^{3} \equiv-a$. Hence $P \equiv 4-4 a(32)$. The case $a \in 4 \mathcal{E}$ corresponds to $X_{1}^{\prime}$ and the case $a \in 4 \mathcal{O}$ corresponds to $X_{2}^{\prime}$. In case (4), by the same argument we obtain $X_{1}$ and $X_{2}$.

In case (5), $b$ and $c$ have the opposite parity. First assume that $b \in \mathcal{E}, c \in \mathcal{O}$. Then $a \in \mathcal{O}, d \in \mathcal{E}$. Let $a+c=4 m$ and $b+d=4 n$, where $n, m \in \mathbb{Z}$. Then

$$
\begin{aligned}
a d+b c & \bmod 8 \equiv a d+(4 m-a)(4 n-d) \equiv 2 a d-4 a n \equiv 2 d-4 n, \\
R & \bmod 8 \equiv d^{2}-a c \equiv 2 d-a(4 m-a) \equiv 2 d+a^{2}-4 a m \equiv 2 d-4 m+1,
\end{aligned}
$$

and hence

$P \quad \bmod 32 \equiv 4(2 d-4 n)+4(2 d-4 m+1) \equiv 16(m+n)+4 \equiv 4(a+b+c+d)+4$.

If $b \in \mathcal{O}, c \in \mathcal{E}$, then by the same argument, we have $P \bmod 32 \equiv 4(a+b+c+d)+4$. The case $a+b+c+d \in 4 \mathcal{E}$ corresponds to $X_{1}^{\prime \prime}$, and the case $a+b+c+d \in 4 \mathcal{O}$ corresponds to $X_{2}^{\prime \prime}$. This completes the proof.

We now give a proof of Proposition 2.4. 
Proof of Proposition 2.4. Let $\tau=\left(\begin{array}{cc}0 & -1 \\ 1 & 0\end{array}\right)$ and $\sigma=\left(\begin{array}{cc}1 & 1 \\ -1 & 0\end{array}\right)$. Then $\{e, \tau, \sigma\}$ is a complete representative of $\Gamma(1) / \Gamma_{0}(2)$, where $e \in \Gamma(1)$ is the identity. In terms of coordinate, the actions of $\tau^{-1}$ and $\sigma^{-1}$ are respectively given by

$$
\begin{aligned}
\tau^{-1}(a, b, c, d) & =(-d, c,-b, a), \\
\sigma^{-1}(a, b, c, d) & =(-d, c+3 d,-b-2 c-3 d, a+b+c+d) .
\end{aligned}
$$

Hence by an easy computation we have $X_{i}^{\prime}=\tau X_{i}$ and $X_{i}^{\prime \prime}=\sigma X_{i}$ for $i=1,2,3$. Thus by Lemma $2.6, L_{1}^{\equiv 4(32)}=\Gamma(1) \cdot X_{1}$ and $L_{1}^{\equiv 20(32)}=\Gamma(1) \cdot\left(X_{2} \sqcup X_{3}\right)$. Let $\gamma \in \Gamma(1)$, $x \in X_{i}$ and $x^{\prime}=\gamma x$. Then, by Lemma 2.1, it is enough to show that $x^{\prime} \in X_{i}$ if and only if $\gamma \in \Gamma_{0}(2)$. Let $x=(a, b, c, d), x^{\prime}=\left(a^{\prime}, b^{\prime}, c^{\prime}, d^{\prime}\right)$ and $\gamma=\left(\begin{array}{ll}p & q \\ r & s\end{array}\right) \in \Gamma(1)$. Then

$$
\left(\begin{array}{l}
a^{\prime} \\
b^{\prime} \\
c^{\prime} \\
d^{\prime}
\end{array}\right)=\left(\begin{array}{cccc}
p^{3} & p^{2} q & p q^{2} & q^{3} \\
3 p^{2} r & p^{2} s+2 p q r & q^{2} r+2 p q s & 3 q^{2} s \\
3 p r^{2} & q r^{2}+2 p r s & p s^{2}+2 q r s & 3 q s^{2} \\
r^{3} & r^{2} s & r s^{2} & s^{3}
\end{array}\right)\left(\begin{array}{l}
a \\
b \\
c \\
d
\end{array}\right)
$$

Let us consider the case $i=1$. Let $x \in X_{1}$. If $\gamma \in \Gamma_{0}(2)$ then we immediately see $x^{\prime} \in X_{1}$. Conversely, assume $x^{\prime} \in X_{1}$. Then $b^{\prime} \equiv$ pra + psb (2). Since $b^{\prime} \in \mathcal{O}$, we have $p \in \mathcal{O}$. Hence $b^{\prime} \equiv r a+s b(2)$ and $d^{\prime} \equiv r a+r s b$ (2). Since $b^{\prime}-d^{\prime} \in \mathcal{O}$, we have $r \in \mathcal{E}$, i.e., $\gamma \in \Gamma_{0}(2)$. Similar arguments work for the cases $i=2,3$, and we have the proposition.

Let

$$
\left(L_{1}^{*}\right)^{\cong l(N)}:=\left\{x \in L_{1}^{*} \mid P(x) / 27 \equiv l(N)\right\} .
$$

Once we have Proposition 2.4, the following is easily obtained.

Proposition 2.7. Let $X_{i}^{*}=X_{i} \cap L_{1}^{*}$ for $i=1,2,3$. Then

$$
\begin{aligned}
\left(L_{1}^{*}\right) \cong-20(32) & =\Gamma(1) \times_{\Gamma_{0}(2)} X_{1}^{*}, \\
\left(L_{1}^{*}\right) \cong-4(32) & =\Gamma(1) \times_{\Gamma_{0}(2)}\left(X_{2}^{*} \sqcup X_{3}^{*}\right) .
\end{aligned}
$$

Proof. For $x \in L_{1}^{*}, P(x) \equiv l(N)$ is equivalent to $P(x) / 27 \equiv 3 l(N)$. Hence $L_{1}^{\equiv l(32)} \cap$ $L_{1}^{*}=\left(L_{1}^{*} \cong 3 l(32)\right.$, and thus

$$
\begin{gathered}
L_{1}^{\equiv 4(32)} \cap L_{1}^{*}=\left(L_{1}^{*}\right)^{\cong 12(32)}=\left(L_{1}^{*}\right)^{\cong-20(32)}, \\
L_{1}^{\equiv 20(32)} \cap L_{1}^{*}=\left(L_{1}^{*}\right)^{\cong 60(32)}=\left(L_{1}^{*}\right)^{\cong-4(32)} .
\end{gathered}
$$

Now the proposition follows from Lemma 2.2.

\section{Proof of the main theorem}

In this section we prove Theorem 1.4. We start with the definition of general partial zeta functions. 
Definition 3.1. For a congruence subgroup $\Gamma$ of $\mathrm{SL}_{2}(\mathbb{Z})$ and a $\Gamma$-invariant subset $X$ of a lattice, we define

$$
\xi_{j}(X, \Gamma, s):=\sum_{\substack{x \in \Gamma \backslash X \\(-1)^{j-1} P(x)>0}} \frac{\left|\Gamma_{x}\right|^{-1}}{|P(x)|^{s}}
$$

We also define

$$
\xi(X, \Gamma, s):=\left(\begin{array}{l}
\xi_{1}(X, \Gamma, s) \\
\xi_{2}(X, \Gamma, s)
\end{array}\right) .
$$

We call them the partial zeta functions for the pair $(X, \Gamma)$.

In this section the complex variable $s$ is always fixed. So we drop $s$ from the notation and write $\xi(X, \Gamma)$ or $\xi[X, \Gamma]$ instead. By definition,

$$
\xi_{i}=\xi\left[L_{i}, \Gamma(1)\right] \quad \text { and } \quad \xi_{i}^{*}=3^{3 s} \xi\left[L_{i}^{*}, \Gamma(1)\right] .
$$

The following proposition is a direct consequence of the definition of the induction.

Proposition 3.2. If $(\Gamma, X)$ is induced from $\left(\Gamma^{\prime}, X^{\prime}\right)$ for a subgroup $\Gamma^{\prime} \subset \Gamma$ and a subset $X^{\prime} \subset X$, then $\xi[X, \Gamma]=\xi\left[X^{\prime}, \Gamma^{\prime}\right]$. Namely, we have

$$
\xi\left[\Gamma \times_{\Gamma^{\prime}} X^{\prime}, \Gamma\right]=\xi\left[X^{\prime}, \Gamma^{\prime}\right] .
$$

Hence Propositions 2.4 and 2.7 respectively imply the following identities.

Proposition 3.3. (1) Define $\xi_{1}^{\equiv l(N)}:=\xi\left[L_{1}^{\equiv l(N)}, \Gamma(1)\right]$. Then

$$
\begin{aligned}
\xi_{1}^{\equiv 4(32)} & =\xi\left[X_{1}, \Gamma_{0}(2)\right], \\
\xi_{1}^{\equiv 20(32)} & =\xi\left[X_{2}, \Gamma_{0}(2)\right]+\xi\left[X_{3}, \Gamma_{0}(2)\right] .
\end{aligned}
$$

(2) Define $\left(\xi_{1}^{*}\right)^{\cong l(N)}:=3^{3 s} \xi\left[\left(L_{1}^{*}\right) \cong l(N), \Gamma(1)\right]$. Then

$$
\begin{aligned}
\left(\xi_{1}^{*}\right) \cong-20(32) & =3^{3 s} \xi\left[X_{1}^{*}, \Gamma_{0}(2)\right], \\
\left(\xi_{1}^{*}\right) \cong-4(32) & =3^{3 s} \xi\left[X_{2}^{*}, \Gamma_{0}(2)\right]+3^{3 s} \xi\left[X_{3}^{*}, \Gamma_{0}(2)\right] .
\end{aligned}
$$

From now on, we study the right hand sides of these identities. We will prove in Theorem 3.8 that they are expressed as linear combinations of the $\xi_{i}$ 's or $\xi_{i}^{*}$ 's.

To study the zeta functions, it is useful to introduce the following action of $G_{\mathbb{Q}}:=$ $\mathrm{GL}_{2}(\mathbb{Q})$ on $V_{\mathbb{Q}}$, which is an extension of the action of $\mathrm{SL}_{2}(\mathbb{Z})$ :

$$
(g \cdot x)(u, v)=\frac{1}{\operatorname{det} g} \cdot x(p u+r v, q u+s v), \quad x \in V_{\mathbb{Q}}, \quad g=\left(\begin{array}{ll}
p & q \\
r & s
\end{array}\right) \in G_{\mathbb{Q}} .
$$

We have the following basic properties of the partial zeta functions. The formula in (2) says that $\xi(X, \Gamma)$ is essentially determined by $X$. In this sense we also say $\xi(X, \Gamma)$ is a partial zeta function for $X$, without referring to $\Gamma$. 
Proposition 3.4. Assume that $X$ is $\Gamma$-invariant.

(1) For $g \in G_{\mathbb{Q}}$, we have $\xi(X, \Gamma)=(\operatorname{det} g)^{2 s} \xi\left(g X, g \Gamma g^{-1}\right)$.

(2) If $\Gamma^{\prime} \subset \Gamma$ is a subgroup, we have $\xi\left(X, \Gamma^{\prime}\right)=\left[\Gamma: \Gamma^{\prime}\right] \xi(X, \Gamma)$.

Proof. (1) follows from $P(g x)=(\operatorname{det} g)^{2} P(x)$ for $g \in G_{\mathbb{Q}}$. We consider $(2)$. Let $X^{ \pm}=$ $\{x \in X \mid \pm P(x)>0\}$. Then

$$
\sum_{y \in \Gamma^{\prime} \backslash X^{ \pm}} \frac{\left|\Gamma_{y}^{\prime}\right|^{-1}}{|P(y)|^{s}}=\sum_{x \in \Gamma \backslash X^{ \pm}} \sum_{y \in \Gamma^{\prime} \backslash \Gamma x} \frac{\left|\Gamma_{y}^{\prime}\right|^{-1}}{|P(y)|^{s}}=\sum_{x \in \Gamma \backslash X^{ \pm}} \frac{\left|\Gamma_{x}\right|^{-1}}{|P(x)|^{s}} \sum_{y \in \Gamma^{\prime} \backslash \Gamma x} \frac{\left|\Gamma_{x}\right|}{\left|\Gamma_{y}^{\prime}\right|} .
$$

Hence (2) follows from the following elementary fact in group theory.

Lemma 3.5. Suppose a group $G$ acts on a set $X$, and $H$ is a finite index subgroup of $G$. Then for $x \in X$ with $\left|G_{x}\right|<\infty$,

$$
\sum_{y \in H \backslash G x} \frac{\left|G_{x}\right|}{\left|H_{y}\right|}=[G: H]
$$

where in the summation of the left hand side, $y$ runs through all the representatives of $H$-orbits in $G x$.

Proof. Consider the canonical bijections $H \backslash G x \simeq H \backslash\left(G / G_{x}\right) \simeq(H \backslash G) / G_{x}$. If $y=$ $g x, g \in G$, then we have $\left|H_{y}\right|=\left|\left(g^{-1} H g \cap G_{x}\right)\right|$ because $H_{y}=H \cap g G_{x} g^{-1}=$ $g\left(g^{-1} H g \cap G_{x}\right) g^{-1}$. Since $g^{-1} H g \cap G_{x}$ is the group of stabilizers of $H g \in H \backslash G$ in $G_{x}$, this implies that $\left|G_{x}\right| /\left|H_{y}\right|$ is equal to the cardinality of the $G_{x}$-orbit of $H g$ in $H \backslash G$. Hence, to sum up using all the representatives on the left hand side is nothing but counting all the elements of the quotient set $H \backslash G$ exactly one time for each, and the result is $[G: H]$.

To study $\xi\left[X_{i}, \Gamma_{0}(2)\right]$, we consider partial zeta functions for the residue classes of $L_{1}$ modulo $2 L_{1}$. Note that each class $(p, q, r, s)+2 L_{1}$ is invariant under the action of $\Gamma(2)$.

Definition 3.6. For $p, q, r, s \in\{0,1\}$, define

$$
\xi_{\text {pqrs }}:=\xi\left[(p, q, r, s)+2 L_{1}, \Gamma(2)\right] .
$$

We also naturally regard $p, q, r, s$ as elements of $\mathbb{Z} / 2 \mathbb{Z}$. It is easy to see that the number of $\Gamma(1)$-orbits in $L_{1} / 2 L_{1}$ is six. Since $\Gamma(2)$ is a normal subgroup of $\Gamma(1)$, by Proposition 3.4 (1), it follows that there are (at most) six different partial zeta functions among all sixteen $\xi_{\text {pqrs }}$. Let us choose $\xi_{0000}, \xi_{0001}, \xi_{0010}, \xi_{0110}, \xi_{0111}, \xi_{1011}$ as the different representatives. Then others are related as

$$
\begin{aligned}
& \xi_{0001}=\xi_{1000}=\xi_{1111}, \quad \xi_{0010}=\xi_{0100}=\xi_{0011}=\xi_{1100}=\xi_{0101}=\xi_{1010}, \\
& \xi_{0111}=\xi_{1110}=\xi_{1001}, \quad \xi_{1011}=\xi_{1101} \text {. }
\end{aligned}
$$

We have the following simple and explicit relations between $\xi_{i}$ 's and $\xi_{p q r s}$ 's. 
Proposition 3.7. (1) We have

$$
\begin{aligned}
6 \xi_{1} & =\xi_{0000}+3 \xi_{0001}+6 \xi_{0010}+\xi_{0110}+3 \xi_{0111}+2 \xi_{1011} \\
6 \xi_{2} & =\xi_{0000}+3 \xi_{0111}, \\
6 \xi_{3} & =\xi_{0000}+\xi_{0110}+2 \xi_{1011}, \\
6 \xi_{4} & =\xi_{0000}+3 \xi_{0001}+\xi_{0110}+3 \xi_{0111}, \\
6 \xi_{5} & =\xi_{0000}+\xi_{0110}, \\
6 \cdot 2^{-4 s} \xi_{1} & =\xi_{0000} .
\end{aligned}
$$

(2) We have

$$
\begin{aligned}
& \xi_{0000}=6 \cdot 2^{-4 s} \xi_{1}, \\
& \xi_{0001}=2\left(\xi_{4}-\xi_{2}-\xi_{5}+2^{-4 s} \xi_{1}\right), \\
& \xi_{0010}=\xi_{1}+\xi_{5}-\xi_{3}-\xi_{4}, \\
& \xi_{0110}=6\left(\xi_{5}-2^{-4 s} \xi_{1}\right), \\
& \xi_{0111}=2\left(\xi_{2}-2^{-4 s} \xi_{1}\right), \\
& \xi_{1011}=3\left(\xi_{3}-\xi_{5}\right) .
\end{aligned}
$$

Proof. Since $[\Gamma(1), \Gamma(2)]=6,6 \xi_{i}=6 \xi\left[L_{i}, \Gamma(1)\right]=\xi\left[L_{i}, \Gamma(2)\right]$. Hence by dividing $L_{i}$ into the disjoint union of residue classes modulo $2 L_{1}$, we have the first five of (1). For example,

$$
6 \xi_{3}=\sum_{\substack{p, q, r, s \in \mathbb{Z} / 2 \mathbb{Z} \\ p+q+r=q+r+s=0}} \xi_{p q r s}=\xi_{0000}+\xi_{0110}+\xi_{1011}+\xi_{1101}=\xi_{0000}+\xi_{0110}+2 \xi_{1011} .
$$

The last one of (1) is because $6 \xi_{1}=\xi\left[L_{1}, \Gamma(2)\right]=2^{4 s} \xi\left[2 L_{1}, \Gamma(2)\right]=2^{4 s} \xi_{0000}$. The formulas in (2) are easily obtained from (1).

Now we will prove the following identities. The authors call these identities kaleidoscopic relations, for this describes their sophisticated symmetry.

\section{Theorem 3.8.}

$$
\begin{aligned}
\xi_{1}^{\equiv 4(32)}= & 3 \cdot 2^{-2 s}\left(\xi_{5}-2^{-4 s} \xi_{1}\right), \\
\xi_{1}^{\equiv 20(32)}= & \left(\xi_{4}-\xi_{2}-\xi_{5}+2^{1-4 s} \xi_{1}\right)+2^{-4 s}\left(\xi_{1}-\xi_{4}-2 \xi_{3}+4 \xi_{5}\right) \\
& -2^{-2 s}\left(\xi_{1}-\xi_{3}-2 \xi_{2}+5 \cdot 2^{-4 s} \xi_{1}\right), \\
\left(\xi_{1}^{*}\right)^{-20(32)}= & 3 \cdot 2^{-2 s}\left(\xi_{4}^{*}-2^{-4 s} \xi_{1}^{*}\right), \\
\left(\xi_{1}^{*}\right)^{\cong-4(32)}= & \left(\xi_{5}^{*}-\xi_{3}^{*}-\xi_{4}^{*}+2^{1-4 s} \xi_{1}^{*}\right)+2^{-4 s}\left(\xi_{1}^{*}-\xi_{5}^{*}-2 \xi_{2}^{*}+4 \xi_{4}^{*}\right) \\
& -2^{-2 s}\left(\xi_{1}^{*}-\xi_{2}^{*}-2 \xi_{3}^{*}+5 \cdot 2^{-4 s} \xi_{1}^{*}\right) .
\end{aligned}
$$

Proof. To prove the first two identities, we compute $\xi\left[X_{i}, \Gamma_{0}(2)\right]$ for $i=1,2,3$. For subsets $A, B, C, D$ of $\mathbb{Z}$, we write $(A, B, C, D)=\left\{(a, b, c, d) \in \mathbb{Z}^{4} \mid a \in A, b \in B\right.$, 
$c \in C, d \in D\}$. Let $g=\left(\begin{array}{cc}1 & 0 \\ 0 & 1 / 2\end{array}\right)$. Then by Proposition 3.4,

$$
\begin{aligned}
\xi\left[(A, B, 2 C, 4 D), \Gamma_{0}(2)\right] & =(\operatorname{det} g)^{2 s} \xi\left[g(A, B, 2 C, 4 D), g \Gamma_{0}(2) g^{-1}\right] \\
& =2^{-2 s} \xi\left[(2 A, B, C, D), \Gamma^{0}(2)\right] .
\end{aligned}
$$

Hence by combining with the identities in Propositions 3.7 (2), we have

$$
\begin{aligned}
\xi\left[X_{1}, \Gamma_{0}(2)\right]= & \xi\left[(\mathbb{Z}, 2 \mathbb{Z}+1,4 \mathbb{Z}+2,8 \mathbb{Z}), \Gamma_{0}(2)\right] \\
= & 2^{-2 s} \xi\left[(2 \mathbb{Z}, 2 \mathbb{Z}+1,2 \mathbb{Z}+1,2 \mathbb{Z}), \Gamma^{0}(2)\right] \\
= & 2^{-2 s}\left[\Gamma^{0}(2), \Gamma(2)\right]^{-1} \cdot \xi_{0110}=3 \cdot 2^{-2 s}\left(\xi_{5}-2^{-4 s} \xi_{1}\right), \\
\xi\left[X_{2}, \Gamma_{0}(2)\right]= & \xi\left[(\mathbb{Z}, 2 \mathbb{Z}+1,4 \mathbb{Z}+2,8 \mathbb{Z}+4), \Gamma_{0}(2)\right] \\
= & 2^{-2 s} \xi\left[(2 \mathbb{Z}, 2 \mathbb{Z}+1,2 \mathbb{Z}+1,2 \mathbb{Z}+1), \Gamma^{0}(2)\right] \\
= & 2^{-2 s}\left[\Gamma^{0}(2): \Gamma(2)\right]^{-1} \cdot \xi_{0111}=2^{-2 s}\left(\xi_{2}-2^{-4 s} \xi_{1}\right), \\
\xi\left[X_{3}, \Gamma_{0}(2)\right]= & \xi\left[(2 \mathbb{Z}+1,2 \mathbb{Z}, 2 \mathbb{Z}, 4 \mathbb{Z}+2), \Gamma_{0}(2)\right] \\
= & \xi\left[(2 \mathbb{Z}+1,2 \mathbb{Z}, 2 \mathbb{Z}, 2 \mathbb{Z}), \Gamma_{0}(2)\right]-\xi\left[(2 \mathbb{Z}+1,2 \mathbb{Z}, 2 \mathbb{Z}, 4 \mathbb{Z}), \Gamma_{0}(2)\right] \\
= & 2^{-1} \xi_{1000}-\xi\left[(\mathbb{Z}, 2 \mathbb{Z}, 2 \mathbb{Z}, 4 \mathbb{Z}), \Gamma_{0}(2)\right]+\xi\left[(2 \mathbb{Z}, 2 \mathbb{Z}, 2 \mathbb{Z}, 4 \mathbb{Z}), \Gamma_{0}(2)\right] \\
= & 2^{-1} \xi_{1000}-2^{-2 s} \xi\left[(2 \mathbb{Z}, 2 \mathbb{Z}, \mathbb{Z}, \mathbb{Z}), \Gamma^{0}(2)\right]+2^{-4 s} \xi\left[(\mathbb{Z}, \mathbb{Z}, \mathbb{Z}, 2 \mathbb{Z}), \Gamma_{0}(2)\right] \\
= & 2^{-1} \xi_{1000}-2^{-1-2 s} \sum_{r, s=0,1} \xi_{00 r s}+2^{-1-4 s} \sum_{p, q, r=0,1} \xi_{p q r 0} \\
= & 2^{-1} \xi_{0001}-2^{-1-2 s}\left(\xi_{0000}+\xi_{0001}+2 \xi_{0010}\right) \\
& +2^{-1-4 s}\left(\xi_{0000}+\xi_{0001}+4 \xi_{0010}+\xi_{0110}+\xi_{0111}\right) \\
= & \left(\xi_{4}-\xi_{2}-\xi_{5}+2^{-4 s} \xi_{1}\right)-2^{-2 s}\left(\xi_{1}-\xi_{2}-\xi_{3}+2^{2-4 s} \xi_{1}\right) \\
& +2^{-4 s}\left(2 \xi_{1}-2 \xi_{3}-\xi_{4}+4 \xi_{5}\right) .
\end{aligned}
$$

Hence the first two identities follows from Proposition 3.3 (1). To prove the other two identities, we compute $\xi\left[X_{i}^{*}, \Gamma_{0}(2)\right]$. By considering the intersection with $L_{1}^{*}$ for each subset in $L_{1}$, a similar proof works. We omit the details.

Remark 3.9. In the previous paper [5], we proved $\xi_{1}^{\equiv 5(8)}=\xi_{2}-2^{-4 s} \xi_{1}$ and $\xi_{1}^{\equiv 1(8)}=$ $\xi_{3}-2^{-4 s} \xi_{1}$. Hence with Theorem 3.8, we have

$$
\begin{aligned}
\xi_{2}= & \xi_{1}^{\equiv 5(8)}+2^{-4 s} \xi_{1}, \\
\xi_{3}= & \xi_{1}^{\equiv 1(8)}+2^{-4 s} \xi_{1}, \\
\left(1-2^{-4 s}\right) \xi_{4}= & \xi_{1}^{\equiv 20(32)}+2^{-2 s}\left(1-2^{-2 s}\right)\left(1+2^{1-4 s}\right) \xi_{1} \\
& +\left(1-2^{1-2 s}\right)\left(\xi_{1}^{\equiv 5(8)}-2^{-2 s} \xi_{1}^{\equiv 1(8)}+3^{-1}\left(1+2^{1-2 s}\right) 2^{2 s} \xi_{1}^{\equiv 4(32)}\right), \\
\xi_{5}= & 3^{-1} 2^{2 s} \xi_{1}^{\equiv 4(32)}+2^{-4 s} \xi_{1} .
\end{aligned}
$$

Thus the coefficients of Dirichlet series $\xi_{2}, \xi_{3}, \xi_{4}, \xi_{5}$ are expressed in terms of coefficients of $\xi_{1}$. We have a similar observation for the dual lattices.

We are now ready to prove Theorem 1.4. 
Proof of Theorem 1.4. We first note that Nakagawa's formula $\xi_{1}^{*}=A \xi_{1}$ in Theorem 1.2 implies $\left(\xi_{1}^{*} \cong-l(N)=A \xi_{1}^{\equiv l(N)}\right.$. The reason for the switch from $l \bmod N$ to $-l \bmod N$ is because $A$ replaces forms of positive discriminants by forms of negative discriminants and vice versa. Recall that we put

$$
\begin{aligned}
\theta & :=\xi_{1}-\xi_{4}-2 \xi_{3}+4 \xi_{5}, \\
\eta & :=2^{2 s}\left(\xi_{4}-\xi_{2}-\xi_{5}+2^{1-4 s} \xi_{1}\right), \\
\theta^{*} & :=2^{2 s}\left(\xi_{5}^{*}-\xi_{3}^{*}-\xi_{4}^{*}+2^{1-4 s} \xi_{1}^{*}\right), \\
\eta^{*} & :=\xi_{1}^{*}-\xi_{5}^{*}-2 \xi_{2}^{*}+4 \xi_{4}^{*},
\end{aligned}
$$

and our goal is to get $\theta^{*}=A \theta$ and $\eta^{*}=A \eta$. By Theorems 1.2, 1.3 and 3.8,

$$
\begin{aligned}
2^{2 s} A \xi_{1}^{\equiv 20(32)} & =A \eta+2^{-2 s} A \theta-A\left(\xi_{1}-\xi_{3}-2 \xi_{2}+5 \cdot 2^{-4 s} \xi_{1}\right) \\
& =A \eta+2^{-2 s} A \theta-\left(\xi_{1}^{*}-\xi_{3}^{*}-2 \xi_{2}^{*}+5 \cdot 2^{-4 s} \xi_{1}^{*}\right) .
\end{aligned}
$$

Therefore since $A \xi_{1}^{\equiv 20(32)}=\left(\xi_{1}^{*} \cong-20(32)\right.$, we have

$$
A \eta+2^{-2 s} A \theta=\xi_{1}^{*}-2 \xi_{2}^{*}-\xi_{3}^{*}+3 \xi_{4}^{*}+2^{1-4 s} \xi_{1}^{*}=\eta^{*}+2^{-2 s} \theta^{*} .
$$

Similarly, from $A \xi_{1}^{\equiv 4(32)}=\left(\xi_{1}^{*} \cong-4(32)\right.$, we have

$$
\theta^{*}+2^{-2 s} \eta^{*}=A\left(\xi_{1}-\xi_{2}-2 \xi_{3}+3 \xi_{5}+2^{1-4 x} \xi_{1}\right)=A \theta+2^{-2 s} A \eta .
$$

These two equalities are equivalent to our desired identities $\theta^{*}=A \theta$ and $\eta^{*}=A \eta$.

\section{Analytic properties of the zeta functions}

In this section we prove Theorems 1.6 and 1.7.

Proof of Theorems 1.6 and 1.7. By [5, Theorem 4.2], we have the functional equation

$$
\xi_{i}(1-s)=2^{2 a_{i} s}\left[L_{1}: L_{i}\right]^{-1} M(s) \xi_{i}^{*}(s),
$$

where

$$
M(s):=\frac{3^{3 s-2}}{2 \pi^{4 s}} \Gamma(s)^{2} \Gamma\left(s-\frac{1}{6}\right) \Gamma\left(s+\frac{1}{6}\right)\left(\begin{array}{cc}
\sin 2 \pi s & \sin \pi s \\
3 \sin \pi s & \sin 2 \pi s
\end{array}\right)
$$

and $a_{1}=0, a_{2}=a_{3}=a_{4}=a_{5}=2$. Hence

$$
\begin{aligned}
\theta(1-s) & =\xi_{1}(1-s)-2 \xi_{3}(1-s)-\xi_{4}(1-s)+4 \xi_{5}(1-s) \\
& =2^{4 s-1} M(s)\left(2^{1-4 s} \xi_{1}^{*}(s)-\xi_{3}^{*}(s)-\xi_{4}^{*}(s)+\xi_{5}^{*}(s)\right) \\
& =2^{2 s-1} M(s) \theta^{*}(s) \\
& =2^{2 s-1} M(s) A \theta(s) .
\end{aligned}
$$

Note that the last equality follows from Theorem 1.4. Similarly, we have

$$
\eta(1-s)=2^{2 s-1} M(s) A \eta(s) .
$$

We put

$$
\Delta(s)=\left(\begin{array}{cc}
\Delta_{+}(s) & 0 \\
0 & \Delta_{-}(s)
\end{array}\right), \quad T=\left(\begin{array}{cc}
\sqrt{3} & 1 \\
\sqrt{3} & -1
\end{array}\right)
$$


Datskovsky and Wright [2] found the following diagonalization of $M(s)$ :

$$
\Delta(1-s) T M(s) A=\Delta(s) T .
$$

Hence

$$
\begin{aligned}
& 2^{1-s} \Delta(1-s) T \theta(1-s)=2^{1-s} \Delta(1-s) T \cdot 2^{2 s-1} M(s) A \theta(s)=2^{s} \Delta(s) T \theta(s), \\
& 2^{1-s} \Delta(1-s) T \eta(1-s)=2^{1-s} \Delta(1-s) T \cdot 2^{2 s-1} M(s) A \eta(s)=2^{s} \Delta(s) T \eta(s),
\end{aligned}
$$

and thus Theorem 1.6 is proved. Finally, Theorem 1.7 immediately follows from the residue formulas of $\xi_{i, j}(s)$ given in [5, Theorem 4.2]. We note that

$$
\frac{\sqrt[3]{2 \pi} \Gamma(1 / 3) \zeta(2 / 3)}{3 \Gamma(2 / 3)}=\frac{\Gamma(1 / 3)^{3}}{2 \pi} \zeta\left(\frac{1}{3}\right) .
$$

Interestingly, the residues at $s=5 / 6$ of $\xi_{1,-}(s), \theta_{-}(s), \xi_{2,-}(s), \xi_{3,-}(s)$ and $\eta_{-}(s)$ vanish. This finishes the proof.

\section{Acknowledgements}

The authors would like to express their gratitude to Manjul Bhargava, Tomoyoshi Ibukiyama and Don Zagier for their valuable discussions and encouragement. The authors are also grateful to Noriyuki Abe for providing a well constructed $\mathrm{C}++$ program to produce experimental data. The first author thanks the Max-Planck-Institut für Mathematik in Bonn for the wonderful working conditions and hospitality. The second author feels indebted to Princeton University Mathematics Department for their support during his stay. The first author is supported by JSPS Grant-in-Aid No. 23540036. The second author is supported by JSPS Grant-in-Aid No. 20740018, No. 20674001 and by JSPS Postdoctoral Fellowships for Research Abroad.

\section{References}

[1] M. Bhargava, Higher composition laws I: A new view on Gauss composition, and quadratic generalizations. Ann. Math. 159 (2004), 217-250.

- Higher composition laws II: On cubic analogues of Gauss composition. Ann. Math. 159 (2004), 865-886

- Higher composition laws III: The parametrization of quartic rings. Ann. Math. 159 (2004), 1329-1360.

- Higher composition laws IV: The parametrization of quintic rings. Ann. Math. 167 (2008), 53-94.

[2] B. Datskovsky and D.J. Wright, The adelic zeta function associated with the space of binary cubic forms II: Local theory, J. Reine Angew. Math. 367 (1986), 27-75.

[3] J. Nakagawa, On the relations among the class numbers of binary cubic forms, Invent. Math. 134 (1998), 101-138.

[4] Y. Ohno, A conjecture on coincidence among the zeta functions associated with the space of binary cubic forms, Amer. J. Math. 119 (1997), 1083-1094.

[5] Y. Ohno, T. Taniguchi and S. Wakatsuki, Relations among Dirichlet series whose coefficients are class numbers of binary cubic forms, Amer. J. Math. 131 (2009), 1525-1541.

[6] M. Sato and T. Shintani, On zeta functions associated with prehomogeneous vector spaces, Ann. of Math. 100 (1974), 131-170. 
[7] T. Shintani, On Dirichlet series whose coefficients are class-numbers of integral binary cubic forms, J. Math. Soc. Japan 24 (1972), 132-188.

[8] T. Taniguchi and F. Thorne, Orbital L-functions for the space of binary cubic forms, Canad. J. Math. 65 (2013), 1320-1383.

Mathematical Institute, Tohoku University, Sendai 980-8578, Japan

E-mail address: ohno@math.tohoku.ac.jp

Department of Mathematics, Graduate School of Science, Kobe University, 1-1, RokKODAi, NADA-KU, Kobe 657-8501, JAPAN

E-mail address: tani@math.kobe-u.ac.jp 